\title{
Alcoolismo na regiáo centro-oeste de Minas Gerais: Perfil SOCIODEMOGRÁFICO, CLÍNICO E DISTRIBUIÇÃo GEOGRÁFICA
}

\author{
Richardson Miranda Machado \\ Moacyr Lobo da Costa Júnior ${ }^{2}$
}

\begin{abstract}
Estudo retrospectivo e exploratório, realizado na Clínica São Bento Menni, com os objetivos: conhecer o perfil sociodemográfico e clínico dos pacientes internados, em decorrência do alcoolismo, e identificar a distribuição geográfica dos casos. Houve predominância do sexo masculino, da faixa etária de 41 a 50 anos, cútis branca, casados, com nível escolar fundamental, trabalhadores autônomos, com diagnósticos de síndrome de dependência, de alta médica hospitalar e de encaminhamentos para os Centros de Atenção Psicossocial. Os municípios de Leandro Ferreira, com 2.955 habitantes, e Araújos, com 7.201 habitantes, tiveram a maior taxa de internação por alcoolismo.
\end{abstract}

Descritores: Alcoolismo; Epidemiologia; Saúde Mental.

\footnotetext{
${ }^{1}$ PhD, Professor Adjunto, Universidade Federal de São João del-Rei, São João del-Rei, MG, Brasil.

2 PhD, Professor Associado, Escola de Enfermagem de Ribeirão Preto, Universidade de São Paulo, Centro Colaborador da OMS para o Desenvolvimento da Pesquisa em Enfermagem, Ribeirão Preto, SP, Brasil.
}

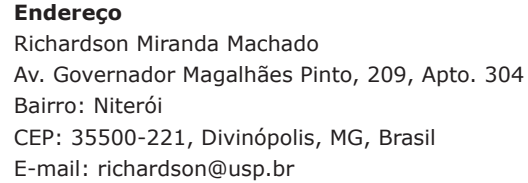




\section{Alcoholism in the midwest region of the state of Minas Gerais: SOCIODEMOGRAPHIC, CLINICAL PROFILES AND GEOGRAPHICAL DISTRIBUTION}

This is a retrospective and exploratory research, conducted at Clínica São Bento Menni aiming at to know the sociodemographic and clinical profiles of inpatients due to alcoholism and to identify the geographic distribution of the cases. There was a predominance of male individuals; from 41 to 50 years old; white; married; with fundamental level; selfemployed; diagnosis of dependence syndrome; discharged from hospitals and referred for Psychosocial Care Centers. The municipalities of Leandro Ferreira with 2955 inhabitants and Araújos with 7201 inhabitants had the highest rate of hospitalization because of alcoholism.

Descriptors: Alcoholism; Epidemiology; Mental Health.

\section{alcoholismo en la región centro este de Minas Gerais: Perfil socio- DEMOGRÁFICO, CLÍNICO Y DISTRIBUCIÓN GEOGRÁFICA}

Estudio retrospectivo y exploratorio, realizado en la Clínica São Bento Menni, con los objetivos: conocer el perfil socio-demográfico y clínico de los pacientes internados en ocurrencia del alcoholismo e identificar la distribución geográfica de los casos. Hubo una predominancia del sexo masculino; de la banda etaria de 41 a 50 años; del cutis blanca; de los casados; del nivel fundamental; de los trabajadores autónomos; de diagnósticos de síndrome de dependencia; de alta médica hospitalaria y de encaminamientos para los Centros de Atención Psicosocial. Los municipios de Leandro Ferreira con 2955 habitantes y Araújos con 7201 habitantes, tuvieron la mayor tasa de internación por alcoholismo.

Descriptores: Alcoholismo; Epidemiología; Salud Mental.

\section{Introdução}

O uso do álcool, na atualidade, é caracterizado como um fenômeno social, representando grave problema à saúde pública mundial, fato que tem despertado a atenção das autoridades de saúde e sanitárias de diversos países.

$\mathrm{O}$ alcoolismo é assim considerado um dos mais graves problemas de saúde pública em todo o mundo. Tendo como principal característica, segundo a Organização Mundial de Saúde (OMS), o estado físico e psíquico alterado, resultante da ingestão de álcool, com o surgimento de reações comportamentais que incluem compulsão pela ingestão contínua ou periódica da substância. Sendo a principal finalidade do usuário experimentar os efeitos físicos causados pela bebida e evitar o desconforto ocasionado pela sua falta ${ }^{(1)}$.

No Brasil, um levantamento nacional evidenciou que $12,3 \%$ das pessoas, com idade entre 12 e 65 anos, são dependentes de álcool, sendo a prevalência de $17,1 \%$ entre a população masculina e $5,7 \%$ na população feminina ${ }^{(2)}$.
Esses percentuais devem ser levados em conta, uma vez que o consumo abusivo acarreta inúmeras consequências negativas para a saúde e para a qualidade de vida do indivíduo e da população, contribuindo para o aparecimento de morbidades que causam a morte e limitações funcionais. Pois, como substância psicoativa, o álcool produz efeito depressor ou euforizante, e seu consumo causa alterações comportamentais (por exemplo, agressividade, conflitos familiares, violência urbana e doméstica), bem como problemas clínicos (por exemplo, hipertensão arterial, gastrite, cirrose, desnutrição) e psíquicos (depressão e distúrbios mentais), contribuindo, também, para a ocorrência de acidentes automobilísticos e absenteísmo laboral ${ }^{(3)}$.

O alcoolismo configura, assim, como um problema para toda a sociedade e principalmente para os pacientes que, a cada dia, inundam os serviços de saúde, da atenção primária até a rede hospitalar, devido ao anseio urgente por 
tratamento e pela cronicidade dos casos. Porém, voltam a naufragar na dependência, devido à falta de estrutura do sistema público de saúde.

Desse modo, como o Sistema Único de Saúde (SUS) tem buscado, de forma técnica, enfrentar o problema do alcoolismo e das internações psiquiátricas, urge a necessidade de se conhecer o perfil sociodemográfico e clínico dos pacientes internados em decorrência do alcoolismo, em 28 anos de atendimento da Clínica São Bento Menni (CSBM), única referência hospitalar para internações psiquiátricas para a região centro-oeste de Minas Gerais, bem como identificar a distribuição geográfica dos casos. Informações essas de fundamental importância para o estabelecimento de medidas de tratamento, vigilância e prevenção mais específicas e eficazes.

Por certo, diante de tais informações, torna-se possível, mediante a reestruturação da psiquiatria, efetivar de vez as ações dos Centros de Atenção Psicossocial (Caps) na região centro-oeste de Minas Gerais, direcionando seus trabalhos e orientando o estabelecimento de políticas públicas de saúde.

\section{Metodologia}

Trata-se de estudo retrospectivo e exploratório, realizado na CSBM, a qual é um hospital psiquiátrico, de cuidado terciário, filantrópico, de médio porte, com capacidade total instalada de 120 leitos. É considerado como o único centro de referência do Sistema Único de Saúde (SUS) para internações psiquiátricas na região centro-oeste de Minas Gerais, atendendo pacientes portadores de transtornos mentais crônicos e agudos para a propedêutica, tratamento psiquiátrico e ambulatorial. Possui clientela diversificada, sendo $85 \%$ dos seus pacientes atendidos pelo SUS, sua grande maioria é proveniente de cidades circunvizinhas à cidade de Divinópolis, Minas Gerais, cidade essa onde está instalada a CSBM, situada no centro-oeste mineiro.

A CSBM desenvolve, por meio do seu Sistema de Internações Hospitalares (SIH), o registro, acompanhamento e arquivo das informações referentes às internações por meio de prontuários, armazenados em seu Setor de Arquivo Médico e Estatístico (Same). Nesse aspecto, instituiu um sistema eletrônico de coleta de dados e registro das internações, a partir de 1995, além de registrar, em formato eletrônico, todos os dados das internações existentes desde 1980, apresentados em formato manuscrito em seu Same.

O presente estudo foi assim realizado a partir da coleta de informações no banco de dados do SIH/CSBM, mediante aprovação do Comitê de Ética em Pesquisa da Universidade de São Paulo (USP), onde, a partir dos dados coletados, foi construído um novo banco de dados com informações acerca dos pacientes acometidos por alcoolismo.

Foram adotados como critérios de inclusão: 1) pacientes internados por alcoolismo na CSBM, no período de 20 de outubro de 1980 (data de inauguração da clínica) a 31 de dezembro de 2008; 2) com permanência na CSBM superior a 24 horas; 3 ) acompanhados desde a admissão até a alta ou transferência. Tendo como critérios de exclusão: pacientes com dados incompletos e/ou errôneos que não puderam ser completados e/ou corrigidos.

Desse modo, na medida em que as informações foram extraídas do $\mathrm{SIH}$, os 50 agrupamentos de informações apresentados pelo item do programa "Access", internações foram transformados em variáveis nas categorias nominais e ordinais, as quais foram inseridas nos softwares Microsoft Excel - versão 2003, e Statistical Package for the Social Sciences (SPSS) - versão 13.0. As informações foram digitadas duas vezes, verificando, dessa maneira, possíveis erros de digitação. Os programas estatísticos permitiram, assim, o tratamento das variáveis coletadas e a apresentação dos resultados de forma descritiva e por meio de figuras.

Dentreas 25variáveisgeradas, três-psicofarmacologia, psicoterapia e terapia ocupacional - foram excluídas dos resultados do presente trabalho. A primeira pela extensão e complexidade dos dados relacionados aos psicofármacos, os quais merecem um trabalho à parte. A segunda e a terceira devido ao não preenchimento dessas informações no SIH da CSBM.

Assim, das 22 variáveis finais, 19 possibilitam resultados sociodemográficos e 3 resultados clínicos. Sendo todas relacionadas à primeira admissão de cada paciente $(\mathrm{n}=2.203)$.

Sendo elas: número de internações, sexo, idade, cútis, estado civil, escolaridade, profissão, cidade de referência, procedência do paciente, acompanhado para a internação, internação voluntária ou involuntária, diagnóstico da $1^{\mathrm{a}}$ internação, convênio, ano da $1^{\mathrm{a}}$ internação, restrição de visitas, restrição de licença, restrição de reinternação, restrição de alta, tempo de duração da $1^{\mathrm{a}}$ internação, tipo de alta, diagnóstico da alta da $1^{\mathrm{a}}$ internação e serviço de referência.

\section{Resultados e Discussão}

No período de 20 de outubro de 1980 (data da inauguração da CSBM) a 31 de dezembro de 2008, foram internados 28.078 pacientes, sendo 2.203 desses pacientes internados em detrimento do alcoolismo.

Em relação ao número de internações, observou-se que o maior número de pacientes alcoólatras $(1796 ; 81,5 \%)$ foi internado uma vez, 303 (13,8\%) foram internados duas vezes e $104(4,7 \%)$ foram internados três vezes ou mais na CSBM.

Quanto ao sexo dos pacientes, houve predominância do sexo masculino com 1.815 (82,4\%) casos. Resultando em uma proporção de 4,6 homens para cada mulher.

A predominância do alcoolismo no sexo masculino está em concordância com a grande maioria dos estudos nacionais e internacionais realizados em serviços psiquiátricos. Nesse sentido, pode-se citar o estudo ${ }^{(4)}$ feito com 8.589 entrevistados, nas 107 cidades do Brasil, com mais de 200 mil habitantes, no qual constatou-se prevalência da dependência de álcool de $17,1 \%$ para 0 sexo masculino e $5,7 \%$ para o feminino. 
Em relação à idade, o maior número de pacientes (715; 32,5\%) concentrou-se na faixa etária de 41 a 50 anos, seguidos dos $667(30,2 \%)$ na faixa etária de 31 a 40 anos, $332(15,1 \%)$ na faixa etária de 51 a 60 anos, 330 (15\%) entre 21 e 30 anos, $94(4,3 \%)$ entre 61 e 70 anos, $34(1,5 \%)$ de 10 a 20 anos e $31(1,4 \%)$ acima de 70 anos. Sendo a idade média de 36,6 anos.

A predominância de pacientes com 41 a 50 anos corresponde ao observado por vários autores que realizaram estudos em serviços hospitalares como a CSBM. O fato de admitir mais pessoas na idade de 41 a 50 anos condiz com o estágio de manifestação dos sintomas crônicos apresentados pelos pacientes em detrimento do uso abusivo de álcool, por vários anos ${ }^{(4)}$.

No que se refere à cútis, a maioria dos pacientes (1.901; 86,3\%) foi classificada como branca, sendo o restante distribuído de forma quase equitativa, com 163 (7,4\%) negros e $133(6 \%)$ pardos. A predominância de pacientes brancos, seguidos de negros, pardos e amarelos se assemelha a outros estudos ${ }^{(5-6)}$.

Ao se avaliar o estado civil, constatou-se pequena predominância de $(1.048 ; 47,6 \%)$ pacientes casados, seguidos de $918(41,7 \%)$ solteiros; $158(7,2 \%)$ divorciados e $79(3,6 \%)$ viúvos.

Em estudo $^{(7)}$ realizado com uma amostra de 300 pacientes alcoolistas, foi constatado que 48,7\% dos pacientes eram casados, tendo esses pacientes apresentado melhor nível de estabilidade e/ou responsabilidade com o tratamento e aderindo por mais tempo.

No que tange ao nível de escolaridade, houve prevalência de $1.073(48,7 \%)$ pacientes com ensino fundamental, seguidos por $816(37 \%)$ com ensino médio, $187(8,5 \%)$ semialfabetizado, 63 (2,9\%) com nível superior incompleto, 39 (1,8\%) com nível superior completo e 25 $(1,1 \%)$ analfabetos.

Os resultados do referido estudo ${ }^{(8)}$ também demonstram que quanto menor a escolaridade e a renda individual maior o risco para a internação por alcoolismo e para a não adesão ao tratamento, o que resultaria em maior tempo de hospitalização, assim como os dados encontrados traduziram a grave situação econômica e social da maioria dos alcoolistas internados nos serviços de saúde pesquisados.

Em relação à profissão dos 2.203 pacientes, foram encontrados 1.172 registros de atividades profissionais diferentes, declaradas no momento da internação. Mediante essa multiplicidade de ocupações não foi possível a comparabilidade das informações acerca da profissão. Assim, foi realizada a codificação das profissões declaradas, seguindo as especificações e agrupamentos da Classificação Brasileira de Ocupações do Ministério do Trabalho.

Assim, constatou-se que a maioria dos pacientes (591; $26,8 \%$ ) era de trabalhadores de serviços (autônomos), vendedores do comércio em lojas e mercados. Seguidos por número significativo de trabalhadores agropecuários, florestais, da caça e pesca $(376 ; 17,1 \%)$ e de trabalhadores da produção de bens e serviços industriais, metalurgia e construção civil $(327 ; 14,8 \%)$.

Sendo também considerável o número de desempregados $(235 ; 10,7 \%)$, aposentado (201; 9,1\%), técnicos de nível médio $(136 ; 6,2 \%)$, trabalhadores de manutenção e reparação $(107 ; 4,9 \%)$, trabalhadores da produção de bens e serviços industriais (têxtil e alimentício) (94; 4,3\%), membros superiores do poder público, dirigentes de organizações de interesse público e de empresas e gerentes $(47 ; 2,1 \%)$, profissionais das ciências e das artes $(44 ; 2,1 \%)$, forças armadas, policiais e bombeiros militares $(24 ; 1,1 \%)$ e trabalhadores de serviços administrativos $(21 ; 1 \%)$.

No que se refere à procedência do paciente para a internação hospitalar, o maior número de pacientes (943; $42,8 \%$ ) era procedente, para a internação na CSBM, das próprias famílias, o que se leva a considerar o fato desse mesmo número de pacientes ser equitativo com o número total de internações pagas por meio de planos de saúde $(530 ; 24 \%)$ e particulares $(413 ; 18,7 \%)$, o que pressupõe maior facilidade para a internação.

É também preocupante o elevado número (455; 20,6\%) de pacientes procedentes dos Centros de Atenção Psicossocial para a internação, fato esse contrário à filosofia desses serviços, criados para acabar com o estigma da hospitalização. Os demais 247 (11,2\%) pacientes eram procedentes de consultório médico particular, $246(11,1 \%)$ do Pronto-Socorro Regional, 162 (7,3\%) por Ordem Judicial e 150 (6,8\%) de Hospital Clínico.

Torna-se, também, oportuno ressaltar o grande número de internações $(1.232 ; 55,9 \%)$ financiadas pelo SUS. Porém, por se tratar de um hospital filantrópico, no qual 80\% dos seus leitos (96 de 120 ativos) são destinados ao SUS, é de se esperar essa prevalência.

Em relação ao paciente apresentar-se acompanhado para a internação, observou-se que a maioria (2.130; $96,7 \%$ ) estava acompanhada no momento da internação. Desse modo, somente 73 (3,3\%) pacientes apresentaram-se desacompanhados. Fato esse que demonstra a necessidade de apoio aos pacientes no enfrentamento da doença e da hospitalização.

No que tange à internação ser voluntária ou involuntária, constatou-se, no decorrer dos anos, queda considerável no número de internações involuntárias. Sendo possível observar declínio importante a partir do ano 1996, período esse marcado pela abertura dos primeiros serviços comunitários de atenção à saúde mental; denominados Núcleos de Atenção Psicossocial (Naps). Ao se avaliar o tempo de internação, também se constatou que os pacientes com internação voluntária apresentaram tempo médio de permanência superior aos pacientes com internação involuntária $(23,1$ vs 21,8 dias). O que evidencia o desejo pela cura do alcoolismo, assim, permanecendo o tempo máximo que for necessário. 


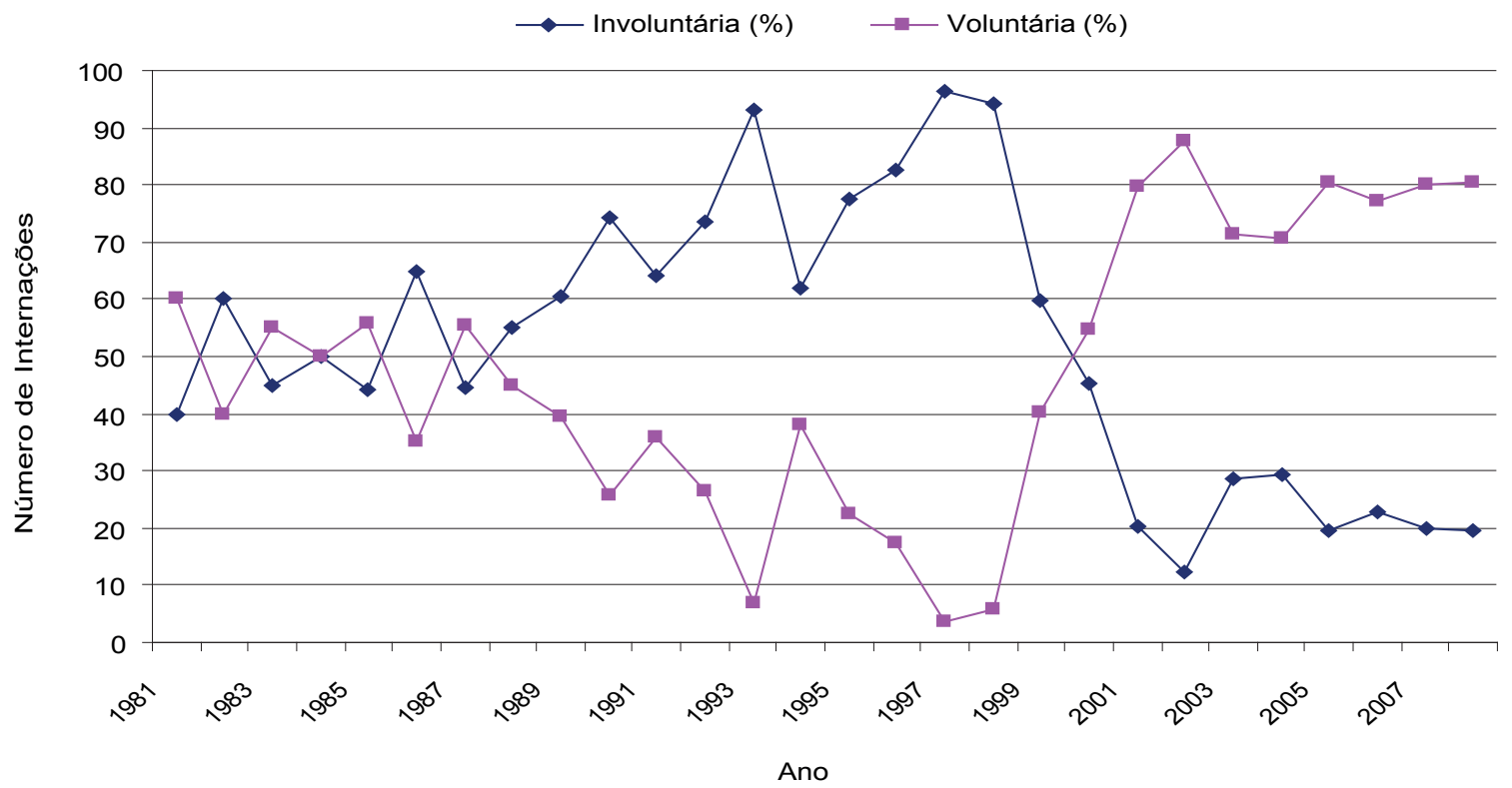

Figura 1 - Distribuição dos pacientes internados por alcoolismo na CSBM, segundo a classificação da internação em voluntária e involuntária. Série Histórica de 1980 a 2008

No período estudado, dos 2.203 pacientes internados, $607(27,6 \%)$ tiveram o diagnóstico da internação baseado na CID-9. É importante ressaltar, nesse período, o elevado número de diagnósticos não especificados $(288 ; 47,4 \%)$ e classificados como outros $(42 ; 6,9 \%)$, o que pressupõe imperícia na condução clínica dos diagnósticos. Torna-se, também, oportuno observar que a maioria dos diagnósticos realizados $(116 ; 19,1 \%)$ refere-se ao quadro de alucinose alcoólica, o que deixa evidente o caráter agudo dos casos. Seguidos por $74(12,1 \%)$ delírium tremens, 34 $(5,6 \%)$ embriaguez patológica, 30 (4,9\%) outra demência alcoólica, $17(2,8 \%)$ psicose alcoólica de Korsakov e 6 (1\%) ciúme alcoólico.

Os outros $1.596(72,4 \%)$ pacientes tiveram seus diagnósticos baseados na CID-10. Cabe ressaltar que, diferente do que foi observado nos diagnósticos baseados na CID-9, houve maior número de casos crônicos de ingestão de álcool, ou seja, 695 (43,5\%) casos de uso de álcool - síndrome de dependência. Os demais pacientes (415; 26,2\%) tiveram diagnóstico de uso de álcool intoxicação aguda, $148(9,2 \%)$ uso de álcool - transtornos psicóticos, $136(8,5 \%)$ uso de álcool e uso de substância psicoativa, 78 (4,9\%) uso de álcool - síndrome (estado) de abstinência, 39 (2,4\%) uso de álcool - síndrome de abstinência com delírio, 29 (1,8\%) uso de álcool - consumo nocivo para a saúde, 24 (1,5\%) uso de álcool - transtorno psicótico residual ou de instalação tardia, $18(1,1 \%)$ uso de álcool - síndrome amnésica - e 14 (0,9\%) uso de álcool outros transtornos mentais e comportamentais.

Ao se estudar os pacientes internados por alcoolismo, foi possível constatar que dos 2.203 casos, 174 (7,9\%) tiveram restrição de visitas, $167(7,6 \%)$ restrição de licenças, 166 (7,5\%) restrição de reinternação e 166 $(7,5 \%)$ restrição de alta. Desse modo, totalizando 673 (30,5\%) pacientes submetidos a algum tipo de medida administrativa e/ou considerada terapêutica.

Ao se avaliar o ano da $1^{\text {a }}$ internação, observa-se, de forma geral, aumento progressivo do número de casos, porém, conforme já constatado anteriormente, é possível identificar queda considerável do número de novas internações por alcoolismo no ano 1996, período de abertura dos primeiros Naps, entretanto, não se verificou redução significativa no ano 2002 (data de criação e início da implantação dos Centros de Atenção Psicossocial), bem como nos anos subsequentes.

Ao se relacionar o ano da $1^{\mathrm{a}}$ internação com o tempo de duração da internação, verifica-se redução importante no tempo médio de internação, de 30 a 40 dias (década de 80), para 20 a 30 dias (década de 90), assim como nos anos seguintes. 


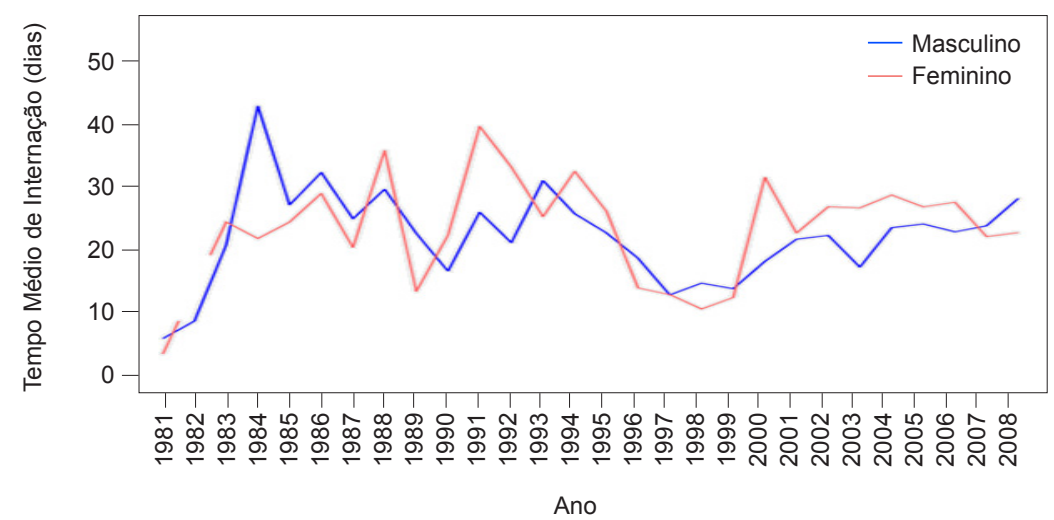

Figura 2 - Distribuição dos pacientes internados por alcoolismo na CSBM, segundo o ano, o tempo de duração da $1^{\mathrm{a}}$ internação e o sexo. Série Histórica de 1980 a 2008

Quando se associou o ano da $1^{\mathrm{a}}$ internação com o tempo de duração da internação e com o sexo dos pacientes, identificou-se inversão na duração do tempo das internações dos pacientes segundo o sexo, onde ocorreu redução do tempo dos pacientes do sexo masculino e aumento no tempo de duração da internação de pacientes do sexo feminino.

Em relação ao tipo de alta, foi prevalente o número de alta médica hospitalar $(1950 ; 88,5 \%)$, o que se leva a considerar como um bom indicador da adesão ao tratamento pelos pacientes, seguidos por 118 (5,3\%) altas a pedido, $53(2,4 \%)$ transferências para clínica médica, $42(1,9 \%)$ altas por abandono, $16(0,7 \%)$ transferências (outros), $12(0,5 \%)$ altas administrativas e $12(0,5 \%)$ altas por evasão.

Dos 2.203 diagnósticos realizados para as internações e para as altas, constatou-se que dos $607(27,6 \%)$ pacientes internados com diagnóstico baseado na CID-9, 567 $(25,7 \%)$ mantiveram o mesmo diagnóstico para a alta. Assim, somente 40 (1,8\%) pacientes tiveram diagnóstico de alta diferente do recebido no momento da internação.

Os $1.596(72,4 \%)$ pacientes que tiveram diagnósticos da internação baseados na CID-10, no momento da alta, somente 1.398 permaneceram com o mesmo diagnóstico. Desse modo, 198 (8,9\%) pacientes tiveram diagnósticos alterados.

No que condiz aos pacientes que tiveram o diagnóstico de alta diferente do recebido para a internação, pode-se verificar que 40 pacientes foram classificados em outra parte, ou seja, com diagnósticos realizados por outros capítulos da CID-9. Desses, 11 (27,5\%) receberam como diagnóstico de alta referente aos quadros psicóticos orgânicos, seguidos por $9(22,5 \%)$ com diagnóstico de psicoses esquizofrênicas, $6(15 \%)$ depressão neurótica, $6(15 \%)$ reação aguda ao stress, $5(12,5 \%)$ estados de ansiedade e 3 (7,5\%) frigidez e impotência.

Foi possível verificar que dos 198 pacientes com diagnósticos de alta, realizados por outros capítulos da CID10 , a maior proporção $(58 ; 29,2 \%)$ recebeu o diagnóstico de esquizofrenia paranoide, seguidos por $40(20,2 \%) \mathrm{com}$ diagnóstico de transtornos mentais orgânicos e 34 (17,1\%) com diagnóstico de psicose não orgânica não especificada, $27(13,6 \%)$ transtorno afetivo bipolar - episódio atual maníaco, 19 (9,6\%) transtornos da alimentação, 12 (6,1\%) personalidade dissocial e 8 (4\%) personalidade histriônica.

Ao se analisar a distribuição dos pacientes, segundo o encaminhamento após a alta (Serviço de Referência), constatou-se que a maioria $(970 ; 44 \%)$ dos pacientes foi referenciada para os Centros de Atenção Psicossocial (Caps), o que deixa transparecer que a instituição hospitalar tem buscado cumprir o disposto na Lei $n^{\circ} 10.216$, de 6/4/2001, a qual normatiza os princípios da Reforma Psiquiátrica e dispõe em seus incisos os Caps como órgãos regulamentadores das internações psiquiátricas. Assim, configurando obrigatoriamente como serviços de referência e contrarreferência para as internações custeadas pelo $\mathrm{SUS}^{(9)}$.

É preocupante, porém, a constatação de que o segundo maior número de encaminhamentos $(513 ; 23,2 \%)$, após a alta, foi para consultório médico particular. $\mathrm{O}$ que leva a tecer considerações quanto à manutenção do sistema mercantilista de assistência à saúde.

Cabe ainda ressaltar o grande número de pacientes $(641 ; 29 \%)$ que teve encaminhamento após a alta (Serviço de Referência), classificado como "outros". Trata-se de uma variedade de serviços, dentre os quais podem ser citados: fazendas de recuperação de drogaditos e alcoólatras, templos religiosos, instituições militares etc., para os quais foi solicitado ao médico assistente o encaminhamento, sendo esse pedido feito pelo próprio paciente e/ou familiares.

Ao se comparar a procedência e o encaminhamento após a alta dos pacientes, é possível verificar que, tendo sido registrados $943(42,8 \%)$ pacientes procedentes da própria família, para a internação, houve somente $9(0,4 \%)$ encaminhamentos após a alta para o seio familiar. Pode-se, assim, pressupor preocupação pelo médico assistente com a manutenção e continuidade do tratamento após a alta.

É oportuno, também, observar que $150 \quad(6,8 \%)$ internações de pacientes procedentes de Hospital Clínico, 
assim como $246(11,1 \%)$ procedentes do Pronto-Socorro Regional, e $37(1,6 \%)$ e 31 (1,4\%) tiveram encaminhamentos após a alta para Hospital Clínico e Pronto-Socorro Regional, respectivamente. Ao se abordar o comprometimento clínico que envolve grande parte dos casos de alcoolismo, é de se considerar a capacidade de resolução de problemas clínicos apresentada pelo hospital psiquiátrico.
Ao se identificar a distribuição geográfica dos casos na região centro-oeste de Minas Gerais, ou seja, a cidade onde o paciente reside e de onde vem encaminhado para a internação, para melhor análise, calculou-se a taxa de internação (por mil habitantes), utilizando-se, para isso, o número de habitantes de cada município ${ }^{(9)}$ pelo número de pacientes internados na CSBM, pertencentes ao mesmo.

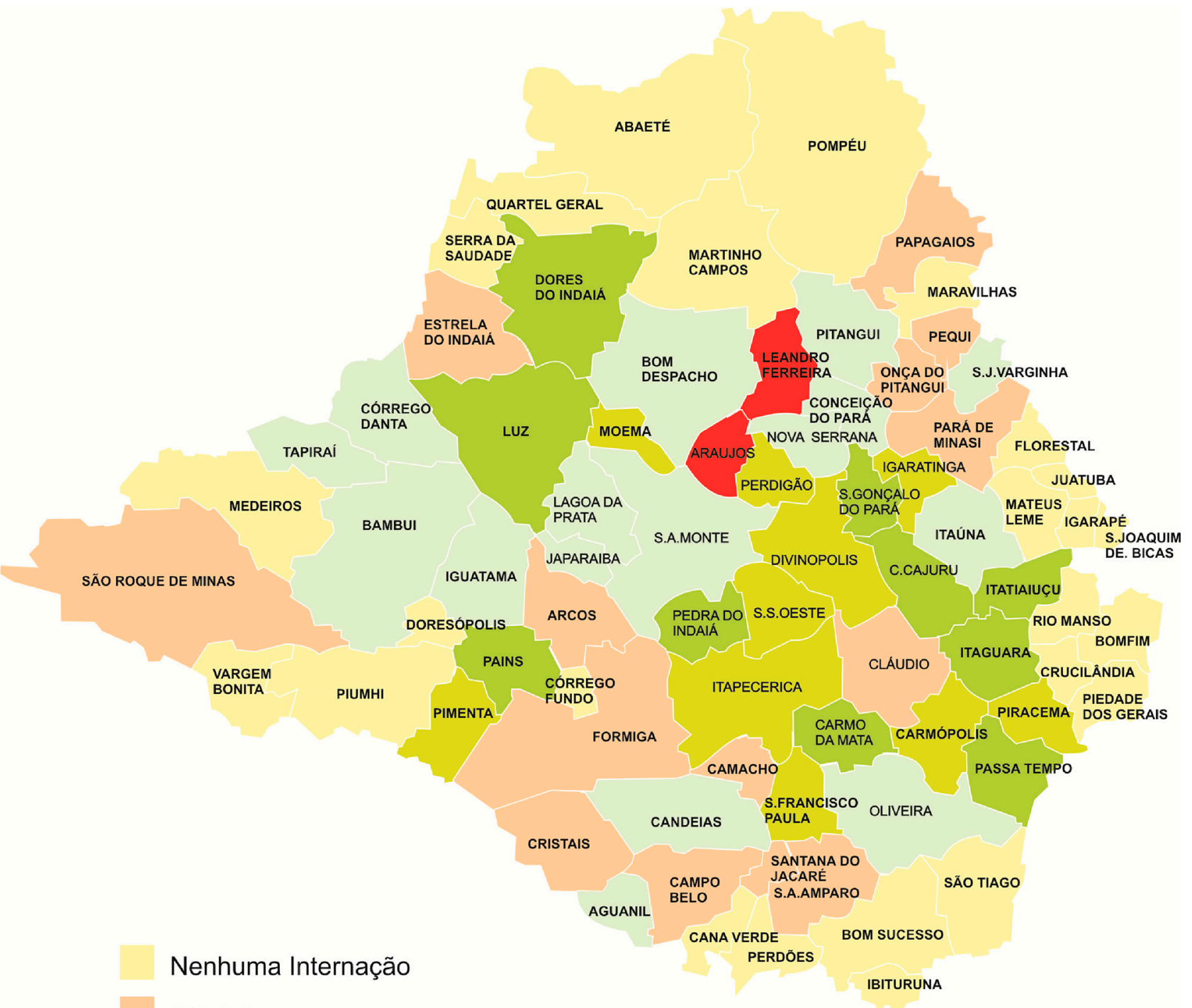

Até 1,0

Acima de 1,0 e menos de 2,0

Acima de 2,0 e menos de 3,0

Acima de 3,0 e menos de 5,0

Acima de 5,0

Figura 3 - Distribuição dos pacientes internados por alcoolismo na CSBM, segundo município de residência na região centro-oeste de Minas Gerais. Série Histórica de 1980 a 2008 
Chamou a atenção o fato de os municípios com menor contingente populacional apresentarem o maior número de internações por alcoolismo. Dados esses compatíveis com outros estudos ${ }^{(10-11)}$, os quais também verificaram predominância do alcoolismo e grande número de internações em municípios de pequeno porte de regiões do interior do país.

Cabe ressaltar que a região centro-oeste de Minas Gerais possui 56 municípios, desses, 9 considerados de médio porte, possuindo entre 30.000 e $80.000 \mathrm{mil}$ habitantes e o município de Divinópolis com mais de 200.000 mil habitantes, tido como de grande porte ${ }^{(12)}$. Nesse aspecto, pode-se inferir que o alto consumo de álcool e o elevado número de internações de pacientes pertencem a municípios de pequeno porte. Assim, estaria relacionado com a pouca oferta de trabalho, de educação profissional, de lazer e cultura.

Chamou a atenção os municípios Leandro Ferreira, com 2.955 habitantes. e Araújos. com 7.201 habitantes, apresentados com maiores taxas de internação por alcoolismo na CSBM/1.000 habitantes, ou seja, acima de 5,0 pacientes a cada 1.000 habitantes, pois são dois dos dez municípios de menor contingente populacional da região centro-oeste de Minas Gerais.

\section{Considerações Finais}

Conforme demonstraram os resultados deste estudo, pode-se considerar que a relação entre o homem e o álcool foi e continua sendo muito conflituosa, gerando, ao final, mais prejuízos do que benefícios. Assim, o consumo de álcool se confirma como grande problema de saúde no Estado de Minas Gerais, em todos os municípios, e a possível relação com fatores pessoais e sociais negativas. Tornando-se, assim, imprescindível a busca contínua por novos saberes acerca do alcoolismo, o que cada vez mais propiciará novo olhar para esse problema. Da mesma forma, suscita dúvidas o fato de haver modificação de diagnósticos em pacientes por ocasião da internação e alta, assim como encaminhamento de pacientes pós-alta para consultórios particulares. Ambas as situações merecem a realização de pesquisas específicas.

Entre as limitações deste estudo ressalta-se a reduzida possibilidade de generalização dos resultados, uma vez que a admissão dos sujeitos obedece, tão somente, a considerações clínicas e administrativas associadas à política assistencial adotada pela CSBM e pelo Sistema Único de Saúde, na região centro-oeste de Minas Gerais. Porém, o desenho utilizado para o seu desenvolvimento permite que ele seja facilmente reproduzido em diferentes contextos assistenciais.

O elevado número de pacientes incluídos neste estudo dificilmente é encontrado em estudos brasileiros e se deve considerar, ainda, que estudos que se desdobram, a partir de dados de Sistemas de Informações já existentes, têm sido cada vez mais valorizados, exatamente por possibilitarem importantes discussões acerca de aspectos relativos à efetividade das práticas assistenciais.

\section{Referências}

1. Secretaria Nacional de Atenção à Saúde. SENAS (BR). Departamento de Ações Programáticas Estratégicas. Manual: Álcool e redução de danos: uma abordagem inovadora para países em transição. Brasília (DF): SENAS; 2008.

2. Centro Brasileiro de Informaçoes sobre Drogas Psicotrópicas (CEBRID). II Levantamento Domiciliar Sobre o Uso de Drogas Psicotrópicas no Brasil: Estudo Envolvendo as 108 Maiores Cidades do País. Sao Paulo (SP): CEBRID; 2006.

3. Heim J, Andrade AG. Efeitos do uso do álcool e das drogas ilícitas no comportamento de adolescentes de risco: uma revisão das publicações científicas entre 1997 e 2007. Rev. psiquiatr. clín. 2009;35(supl 1):61-4.

4. Galduroz JCF, Caetano R. Epidemiologia do uso de álcool no Brasil. Rev. Bras. Psiquiatr. 2009;26(1):3-6.

5. Gigliotti A, Bessa MA. Síndrome de Dependência do Álcool: critérios diagnósticos. Rev. Bras. Psiquiatr. 2009;26(1):11-3.

6. Meloni JN, Laranjeira R. Custo social e de saúde do consumo do álcool. Rev. Bras. Psiquiatr. 2004;26(1):7-10. 7. Seifert J. The development of alcohol dependence in Germany results from a multicenter study. Journal Psychiatr Prax. 2004;31(2):83-9.

8. Ribeiro MS. Fatores associados à adesão a um programa de tratamento de alcoolistas. J. Bras. Psiquiatr 2008;57(3):203-11.

9. Lei n. 10.216 (BR). 06 de abril de 2001. Dispõe sobre a proteção e os direitos das pessoas portadores de transtornos mentais e redireciona o modelo assistencial em saúde mental. Diário Oficial da União; 09 de Abr. 2001.

10. Mateos R, Páramo M, Carrera I, Rodrígues-López A. Alcohol consumption in a southern European region (Galicia, Spain). Subst Use Misuse. 2002;37(14):1957-76. 11. Gallassi AD, Alvarenga PG, Andrade AG, Couttolenc BF. Custos dos problemas causados pelo abuso do álcool. Rev. psiquiatr. Clín. 2008;35(suppl 1):25-30.

12. Ministério do Planejamento Orçamento e Gestão (BR). Distribuição Geográfica, Espacial e Delimitação Territorial. Brasília (DF): Ministério do Planejamento; 2008. 